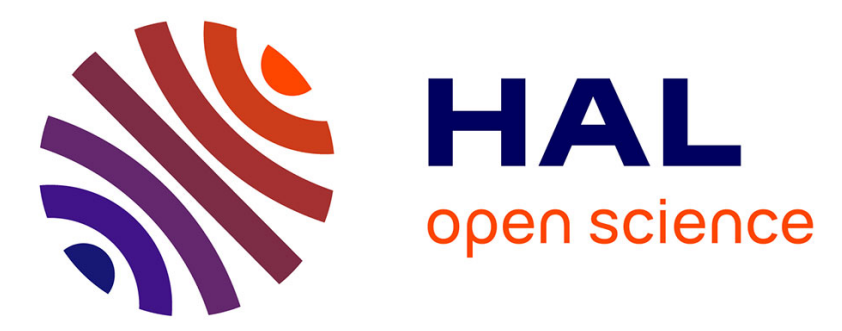

\title{
Digital health interventions to help living with cancer: A systematic review of participants' engagement and psychosocial effects
}

Geraldine Escriva-Boulley, Tanguy Leroy, Camille Bernetière, Françoise Paquienseguy, Orélie Desfriches-Doria, Marie Préau

\section{To cite this version:}

Geraldine Escriva-Boulley, Tanguy Leroy, Camille Bernetière, Françoise Paquienseguy, Orélie Desfriches-Doria, et al.. Digital health interventions to help living with cancer: A systematic review of participants' engagement and psychosocial effects. Psycho-Oncology, 2018, 27 (12), pp.2677-2686. 10.1002/pon.4867 . hal-03230475

\section{HAL Id: hal-03230475 \\ https://hal.science/hal-03230475}

Submitted on 19 May 2021

HAL is a multi-disciplinary open access archive for the deposit and dissemination of scientific research documents, whether they are published or not. The documents may come from teaching and research institutions in France or abroad, or from public or private research centers.
L'archive ouverte pluridisciplinaire HAL, est destinée au dépôt et à la diffusion de documents scientifiques de niveau recherche, publiés ou non, émanant des établissements d'enseignement et de recherche français ou étrangers, des laboratoires publics ou privés. 
archives-ouvertes

\section{Digital health interventions to help living with cancer: A systematic review of participants' engagement and psychosocial effects}

Geraldine Escriva-Boulley, Tanguy Leroy, Camille Bernetière, Françoise Paquienseguy, Orélie Desfriches-Doria, Marie Préau

\section{To cite this version:}

Geraldine Escriva-Boulley, Tanguy Leroy, Camille Bernetière, Françoise Paquienseguy, Orélie Desfriches-Doria, et al.. Digital health interventions to help living with cancer: A systematic review of participants' engagement and psychosocial effects. Psycho-Oncology, Wiley, 2018, 27 (12), pp.2677-2686. 10.1002/pon.4867 . hal-03230475

\section{HAL Id: hal-03230475 \\ https://hal.archives-ouvertes.fr/hal-03230475}

Submitted on 19 May 2021

HAL is a multi-disciplinary open access archive for the deposit and dissemination of scientific research documents, whether they are published or not. The documents may come from teaching and research institutions in France or abroad, or from public or private research centers.
L'archive ouverte pluridisciplinaire HAL, est destinée au dépôt et à la diffusion de documents scientifiques de niveau recherche, publiés ou non, émanant des établissements d'enseignement et de recherche français ou étrangers, des laboratoires publics ou privés. 


\title{
Digital health interventions to help living with cancer: A systematic review of participants' engagement and psychosocial effects
}

\author{
Géraldine Escriva Boulley ${ }^{1}$ ○ | Tanguy Leroy ${ }^{1}$ | Camille Bernetière ${ }^{2}$ | \\ Françoise Paquienseguy ${ }^{2}$ | Orélie Desfriches-Doria ${ }^{2}$ | Marie Préau ${ }^{1}$
}

\author{
${ }^{1}$ Laboratory: Social Psychology Research \\ Group [Groupe de Recherche en Psychologie \\ Sociale] (EA 4163), Psychology Institute \\ [Institut de Psychologie]-Lyon 2 University \\ [Université Lyon 2], Bron, France \\ ${ }^{2}$ Laboratory: Information and Communication \\ Sciences Lyon Research Team [Équipe de \\ Recherche de Lyon en Sciences de \\ l'Information et de la Communication] [EA \\ 4147), Institute of Human Sciences [Institut \\ des Sciences de l'Homme]-Lyon University \\ [Université de Lyon], Lyon. France \\ Correspondence \\ Géraldine Escriva Boulley, Groupe de \\ Recherche en Psychologie Sociale (EA 4163), \\ Institut de Psychologie-Université Lyon 2. \\ Bâtiment $V$ (1er étage), 5 avenue $P$. \\ Mendès-France, 69676 Bron, France. \\ Email: escrivageraldine@gmail.com \\ Funding information \\ Metropole de Lyon
}

\begin{abstract}
Objective: Digital health interventions (DI) open the possibility for cancer patients and survivors to manage the disease and its side effects when they return home after treatment. This study aims to highlight the components of DI, investigate patient engagement with DI, and explore the effects of DI on psychosocial variables.

Methods: In September 2017, we performed a systematic review of studies focusing on DI which target cancer patients or survivors.

Results: A total of 29 articles ( 24 studies) were reviewed. There was considerable heterogeneity in study methods, in outcome definitions. in measures for engagement with DI and in psychosocial variables assessed. Results from the studies showed a high level of engagement. Self-efficacy, psychological symptoms, and quality of life were the most commonly assessed psychosocial variables. However, results for the effect of DI on psychosocial variables were inconsistent. Regarding pain management, results were in line with what one would expect.

Conclusions: The present review showed that despite the heterogeneity in the studies assessed and inconsistent results, DI may constitute an excellent means to help cancer patients and survivors cope better with the disease and with treatment side effects, as they can improve self-management and wellbeing. In order to acquire a greater understanding of the mechanisms underlying cancer patients'/survivors' psychological and behavioral changes in terms of adopting DI. direct comparison between studies is needed. However, this can only come about if methodological and conceptual standardization of DI is implemented.

KEYWORDS

cancer patients, cancer survivors, e-health, interventions in oncology, m-health, non-randomized study interventions. randomized control trials
\end{abstract}

\section{1 | BACKGROUND}

In addition to physical issues, cancer patients (ie, on treatment) and cancer survivors (ie, post-treatment) have to deal with several adverse psychological effects (eg, fatigue, anxiety) which negatively affect health-related quality of life. ${ }^{1-3}$ Most patients and survivors may not see a health professional for a relatively long time after returning home from hospital. They therefore need a degree of autonomy in managing disease symptoms and side effects due to disease and treatment. To achieve this, patients require skills and knowledge about how to find and use information regarding their health situation. ${ }^{4.5}$ This greater autonomy may lead to a faster return to daily activity 
and work ${ }^{6,7}$ and therefore greater psychological wellbeing. Programs and interventions using digital technology (ie, digital health interventions-DI) may be an effective way to reach these desired outcomes.

Digital health interventions are becoming increasingly ubiquitous in primary prevention and detection, diagnosis, treatment, and in the management of associated side effects. ${ }^{8}$ They can be delivered through digital tools (D-tools) including computers, websites, mobile phones, smartphone applications (apps), and wearable devices. The major aim of DI is to foster and support behavioral change. Although the potential for DI to help patients manage cancer is generally accepted, few tailor-made cancer-focused DI currently exist when compared with contexts such as smoking, ${ }^{9}$ alcohol consumption, ${ }^{10}$ weight management, ${ }^{11}$ physical activity (PA), ${ }^{12}$ and self-management of chronic conditions. ${ }^{13}$

Nevertheless, several DI for cancer are under development. Understanding the engagement of patients and survivors in existing $\mathrm{DI}$ is important when developing DI. Engagement can be conceptualized as a multidimensional construct including behavioral dimensions (eg, amount, range, duration, frequency) and subjective experiences that include cognitive and emotional states, such as attention, interest, and affect. ${ }^{14}$ It is assumed that the greater the engagement with DI, the greater their effectiveness. ${ }^{14,15}$ For example, a positive association between DI engagement and smoking cessation ${ }^{16}$ and weight loss ${ }^{17}$ has aiready been observed. Accordingly, to evaluate DI effectiveness in the cancer setting, patients and survivors' engagement with DI needs to be assessed.

Reviews of DI targeting cancer are scarce. Some highlighted that DI could be useful for individuals during cancer treatment and afterwards, especially in terms of information, follow-up planning, and managing side effects. ${ }^{18.19}$ Three others showed that DI could have positive effects on adult cancer survivors' psychosocial variables (eg, wellbeing, stress) and behaviors. ${ }^{20-23}$ Two of the three focused exclusively on DI based on websites. The third tried to target mobile phones and apps but found no article which used these D-tools. ${ }^{22}$

Two reviews ${ }^{18,24}$ found that engagement with DI was high. However, this variable was only examined in reviews targeting adolescents and young adults. All the cancer-based reviews cited ${ }^{18-24}$ highlighted that $\mathrm{DI}$ are mostly multi-component in nature, and that there was a great deal of heterogeneity in the protocols and outcomes measured in cancer-related DI studies they reviewed. It is worth noting that both participants' engagement and psychosocial variables were rarely assessed at the same time in the DI studies and were never examined at the same time in reviews.

Given that DI can be seen as a complement to traditional care (ie, provided by health institutions, health professionals), improving knowledge about the components of $\mathrm{DI}$, engagement with $\mathrm{DI}$, and mechanisms through which DI exert their effects is a challenge which can only be addressed by implementing more research. To design effective DI, there is a need to increase knowledge about the DI components that are usually used in the cancer setting, and what the most popular ones are (ie, what components generate the greatest participant engagement). It is also important to examine the relationships between $\mathrm{DI}$ and participant engagement and wellbeing, to ensure that $\mathrm{DI}$ are a viable solution in the cancer setting. To understand the effectiveness of $\mathrm{DI}$, it is necessary to analyze results from comparisons between a treatment group (TG) and a control group (CG), or from comparisons between pre- and post-tests. The purpose of this systematic review is to examine the literature on DI designed to help cancer patients and survivors live with their disease, and to explore their effects on disease and treatment side effects, and on patients' psychological wellbeing. To do this, our review first identifies the components of cancer-related DI. It then investigates and disentangles the elements of engagement with DI and/or associated D-tools, identifies the psychosocial variables targeted (as an indicator of psychological wellbeing) by $\mathrm{DI}$, and highlights the effects of $\mathrm{DI}$ on these variables.

\section{2 | METHODS}

\section{1 | Search strategy}

This systematic review was conducted in accordance with the AMSTAR checklist (A MeaSurement Tool to Assess systematic Reviews, $\left.{ }^{25,26}\right)$. A comprehensive literature search was conducted to identify scientific articles that analyzed DI targeting cancer patients or survivors. A systematic search of electronic databases, including MEDLINE, PsychINFO, Psycharticle, and Sport Discuss was, conducted to identify articles published before September 2017. The following search terms were used in combination: digital health, mobile health, m-health, mhealth, mobile application, mobile apps, cancer, intervention, program, treatment, therapy, and strategy.

\subsection{Eligibility criteria}

To be considered for inciusion, articles were required to meet the following criteria: (1) reviewed and published in English, (2) tested one or more DI, (3) assessed psychosocial variables or engagement with DI (4) presented DI focused on helping patients or survivors to autonomously manage their health condition on a daily basis (eg, providing strategies, training), (5) presented a main study, a pilot study, or an exploratory study, and, (6) presented a study which had one of the following design types: randomized controlled trial (RCT), cross-sectional study or pretest-posttest study. Articles detailing studies which used RCT and non-randomized study interventions were included to compensate for the currently small number of published cancer-based DI studies, and to allow a comprehensive analysis of the effects of D to be carried out

Studies were excluded when (1) the DI presented aimed at preventing or detecting cancer, (2) participants did not actually use D-tools, (3) psychosocial variables, or engagement with DI were not assessed, (4) the DI presented aimed at improving self-care (eg, medication adherence, going to medical visits), (5) the DI presented targeted health care professionals or providers, and (6) there were reporting issues (eg, only the abstract was available, systematic reviews, meta-analyses).

\section{3 | Data selection and extraction}

Preliminary screening was conducted based on titles and abstracts. Full-text articles were obtained for all abstracts which initially met the inclusion criteria for further evaluation, and for articles that could 
not be rejected with certainty. The reference lists of studies meeting the eligibility criteria were screened in order to potentially include additional relevant studies. Two of the authors independently screened the full-text copy of every article in accordance with the inclusion and exclusion criteria. Any disagreement was resolved by discussion. The agreement rate between the two reviewers was $91 \%$ (985/1083 studies). After discussion, the following details for each study were systematically extracted: first author, year of publication. country of origin, study design, objective, total sample size, intervention period, characteristics of the population lage, gender, type/site of cancer), type of D-tools used, outcome measures, measurement tool, intervention component, and results. The process of data selection is outlined in Figure 1.

\section{4 | Risk of bias assessment}

The risk of bias for RCT studies which were included in this review was assessed using the Cochrane Collaboration's tool for assessing risk of bias. ${ }^{27}$ Risk of bias was classified (low, unclear, or high risk of bias) based on the Cochrane risk of bias tool (Table 1 in supplementary material). For non-RCT studies, the risk of bias was assessed using the Risk of Bias in Non-randomized Studies-of Interventions (ROBINS-1 ${ }^{28}$;) tool. Risk of bias was categorized (low, moderate, serious, or critical risk, or no information) based on the ROBINS-1 tool (Table 2 in Supporting Information).

\section{3 | RESULTS}

\section{1 | Study selection}

The literature search yielded a total of 1110 potential eligible articles (Figure 1). After completely reviewing the 84 full-text articles selected after screening abstracts and titles, 29 articles were eligible for inclusion in the systematic review. The main reasons for exclusion are reported in Figure 1.

\subsection{Study characteristics}

Selected articles were published between 2001 and 2017. Twenty-nine articles were included corresponding to 24 studies. More than one article was possible for the same study due to changes in study design (protocol, sample size, etc.). Of the 24 studies, 14 were conducted in USA, two in Netherlands, United Kingdom, and Canada, and one in Australia, Sweden, Norway, and Korea. Twelve articles presented exploratory or pilot studies, 16 presented main studies, and one presented a usability study. Study design included 15 randomized control trials, six posttests (cross-sectional), five pre-posttests, and three quasi-randomized/non-randomized control trials.

Among the 29 articles, 14 referred to studies where patients were undergoing treatment and 15 on cancer survivors. The mean number of participants across the 29 articles was 105.69 (range: 4-492), of which $81.24 \%$ were female. Age ranged between 8 and 80 years.

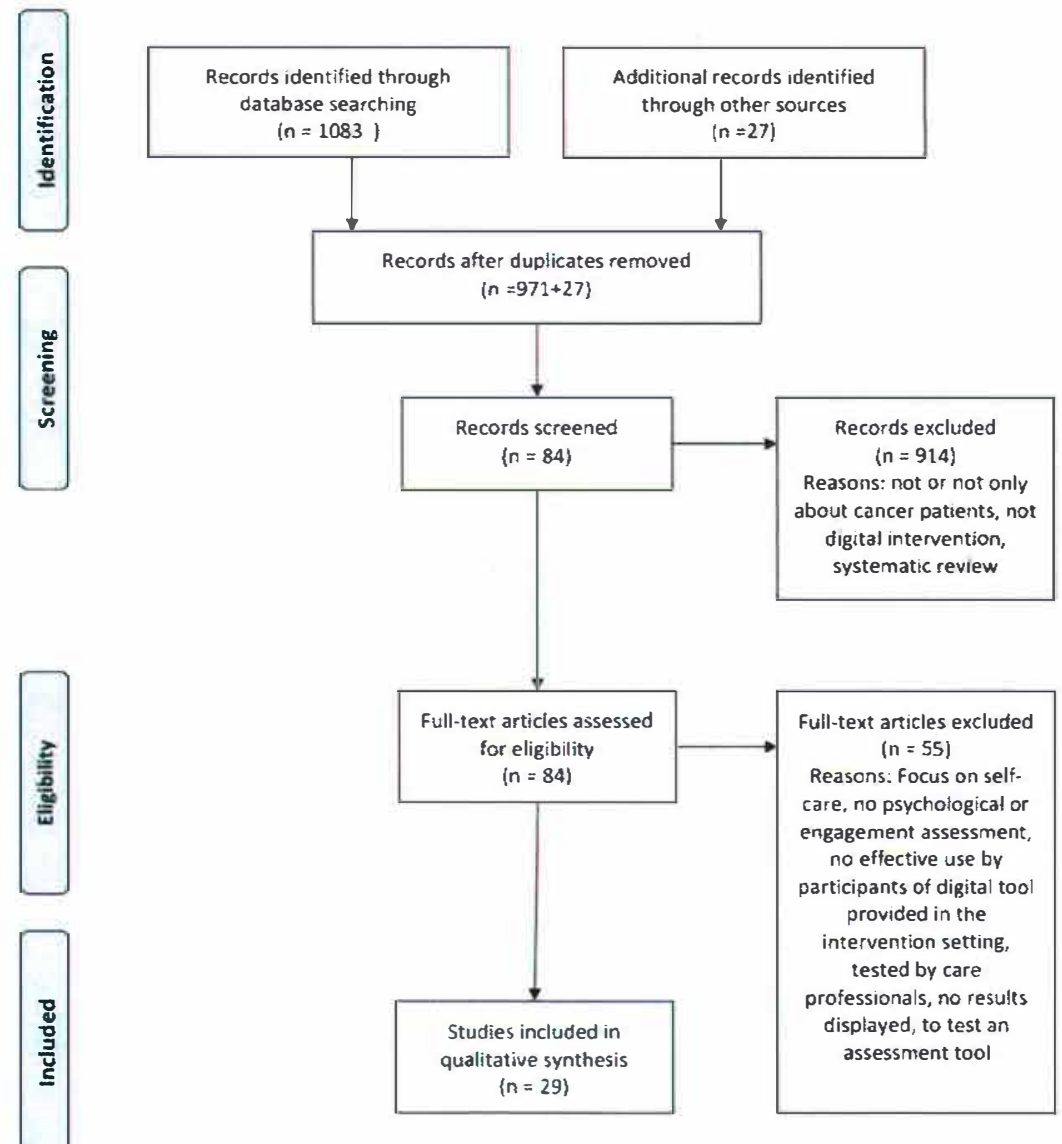


Most of these articles targeted breast cancer only (38\%) or breast cancer and other types of cancer.

Digital health intervention duration varied in the different studies between 10 days and more than 6 months. In two studies, DI was used "for as long as needed." 29.30 Nine studies reported a postintervention follow-up measurement. ${ }^{30-38}$

Twelve of the 24 studies were theory-based. Theories used included Bandura's theories $(\mathrm{DI})^{31,34,36,39-41}:$; Theory $\left.{ }^{42,43}:\right)$, gate control theory, ${ }^{29,44.45}$ and goal-setting theory. ${ }^{46.47}$ The following theories were cited as theoretical frameworks but were not in fact used in the DI discussed: the trans-theoretical model, ${ }^{48,49}$ planned-behavior theory, ${ }^{41.50}$ self-determination theory, ${ }^{51.52}$ the social contextual model, ${ }^{53.54}$ and self-regulation theory. ${ }^{55.56}$ Data from the 24 studies are summarized in the Supporting Information (see Table 3 for patients and Table 4 for survivors).

\section{4 | SYSTEMATIC REVIEW}

\subsection{Intervention salient components}

Study characteristics were heterogeneous, especially in terms of the DI tested and their components. Different D-tools were used in DI such as live videoconferencing, ${ }^{29.44}$ online interactive workshops, ${ }^{40}$ websites $^{33,34.38,48.55 .57-59}$ including social networks for synchronous or asynchronous communication, ${ }^{35.51 .60,61}$ text messages, ${ }^{31.36 .37}$ automated voice response, ${ }^{62}$ PA tracking devices or Wi-Fi weighing scales associated with an app, ${ }^{51.53 .55 .63}$ and apps for computer laptops, smartphones, and/or tablets. ${ }^{30.33 .39 .41 .46,53,64-67}$ The various components of DI aimed at helping cancer patients and survivors cope better with their disease were classified into one of two categories: psychosocial components and design components.

Psychosocial components included: education and information, psychological support, and strategies to optimize wellbeing.

\subsection{1 | Education and information}

Some DI provided education and information about disease side effects, symptoms (eg, ${ }^{35,39}$ ) or pain management ${ }^{29.65}$ and ways to cope with the consequences of cancer $\left(e g,{ }^{62,64}\right)$. For instance, they explained (1) which activities to perform and how to perform them, (2) potential risks, (3) when to contact a physician following symptom onset, and (4) links to trusted websites. ${ }^{59}$ In addition to this kind of information, some DI also provided information about weight management and/or PA (eg, $\left.{ }^{34.48 .55}\right)$. For instance, participants received guidelines about PA, education about the benefits of PA, ${ }^{34}$ and details about common myths related to PA. ${ }^{48}$ The information provided by DI came as much from trusted sources $\left(\mathrm{eg}^{35.58}\right)$ as it did from other patients. $^{60}$

\subsection{2 | Psychological support}

With DI based on social networks or website accounts, patients could read about other patients' experiences with cancer as well as share their own experience. ${ }^{57}$ They could also receive or give support, information, and advice $\left(\mathrm{eg},{ }^{59,60}\right)$. Contrary to the networks proposed to adults $\left(e g,{ }^{45.46,57}\right)$, which were specifically created for the purpose of the studies analyzed, the social network proposed to adolescents and young adults ${ }^{51}$ was pre-existing and well-known (Facebook). Furthermore, to favor psychological support, some DI included encouragement in the form of reinforcement for current behaviors, ${ }^{55}$ the use of badges, ${ }^{51}$ or messages with congratulations. ${ }^{48}$

For some DI, patients and survivors could call or be contacted by the researcher or health care professionals. The purpose of these calls was to help them prepare questions for their physician, set goals, and receive advice on how to implement behavior changing techniques $\left(\mathrm{eg}^{34,51}\right)$. Communication could be synchronous or asynchronous. DI could also provide virtual relationships using videoconferences $\left(\mathrm{eg}^{29.44}\right)$ or text messaging. ${ }^{36}$ One virtual relationship provided to children consisted in a $3 D$ avatar that guided the patients throughout the program using both speech and animation components. $^{39}$

\subsubsection{Strategies to optimize wellbeing}

DI could also include components which dealt with treatment consequences and optimize wellbeing. They provided patients with strategies to manage symptoms (eg, pain, lymphedema symptoms) including guided imagery, muscle relaxation, diaphragmatic breathing, and mindfulness $\left(\mathrm{eg}^{33,39}\right)$. To do this, training was proposed through the DI to develop cognitive and behavioral skills for pain and symptoms management. ${ }^{39}$ With respect to PA, participants were also given tips, instructions, and videos to illustrate exercises $\left(e g,{ }^{63.66}\right)$. The aim was to avoid relapse and to overcome barriers to PA. ${ }^{48}$ Patients also learned how to schedule activity to be productive and active while avoiding increased symptoms due to excess activity.

The second category of DI components, design components, included: tailoring interventions, giving follow-up and feedback, and providing functionalities which would aid the patients in their everyday lives.

\subsection{4 | Tailored interventions}

Some D-tools were tailored to individual patient data. Upon reporting their symptoms patients immediately received both an evaluation of their symptoms and tailored recommended strategies $\left(\mathrm{eg},{ }^{33.59}\right)$. For instance, based on self-reported symptoms, the D-tool used in the DI might display the appropriate subset of self-management activities which patients could choose from. ${ }^{59}$

In order to tailor the body weight and PA programs, participants were asked for information (eg, energy balance, PA preferences, recovery stage) $\left(\mathrm{eg}^{66,67}\right)$. Based on this information, individualized exercise programs were designed $\left(\mathrm{eg},{ }^{40.48}\right)$. In some DI, body weight and PA goals could be negotiated or set by participants (eg, $\left.{ }^{46.66}\right)$.

\subsection{5 | Follow-up and feedback}

With these DI, participants were asked to report or track self-related symptoms, their mood $\left(\mathrm{eg},{ }^{58.59}\right), \mathrm{PA}^{35.61}$ and their weight ${ }^{41}$ or, to post an action plan for PA. ${ }^{40}$ These $\mathrm{DI}$ offered patients the possibility to see changes over time through visual displays. ${ }^{51.67}$ Based on this data, participants could obtain real-time feedback on their behavior. ${ }^{41}$ 


\subsubsection{Functionalities}

Some DI provided several functionalities to help patients in everyday life and cope better with side effects. For instance, they could use a diary to write questions for future medical visits, record symptoms, ${ }^{39}$ use a calendar feature to record each exercise session, side effects, and share their satisfaction with the treatment. ${ }^{65}$ Additionally, push notifications or phone calls were used to increase engagement and user retention $\left(\mathrm{eg},{ }^{41,66}\right)$. They were also used as a motivational reminder and to reward adherence. ${ }^{41,51}$

\subsection{Participant engagement}

\subsection{1 | Measurement tools}

Engagement was measured using both self-reported questionnaires and interviews. Measurement tools were designed or chosen by authors for the purpose of the given study $\left(\mathrm{eg}^{35,44}\right)$ or were assessed with a measurement tool used or validated in a previous study $\left(\mathrm{eg},{ }^{33,48}\right)$

\subsubsection{Variables assessed to evaluate engagement}

Participant engagement with DI was evaluated in several different ways and using several variables as follows: feasibility $\left(\mathrm{eg},{ }^{29.35}\right)$, acceptability $\left(\mathrm{eg},{ }^{34,55}\right)$, usability $\left(\mathrm{eg},{ }^{33,34}\right)$, satisfaction $\left(\mathrm{eg},{ }^{48,55}\right)$, usage $^{34}$ user feedback, compliance, and adherence rate $\left(e g,{ }^{48,57}\right)$. In some cases, participants were also asked in a specific question whether they would recommend the D-tool they were using $\left(e g,{ }^{63,67}\right)$.

The questionnaires used to assess these variables were heterogeneous across studies. For instance, "feasibility" was assessed in terms of (1) completion or not of an intervention session, (2) attrition or adherence rate $\left\{\mathrm{eg}^{35,40}\right),(3)$ the number of assessments or text messages delivered and replied to, ${ }^{36}$ (4) the efficacy of the website on symptoms, symptoms distress, and quality of life, ${ }^{33}$ and (5) the number of eligible participants who enrolled in the study. ${ }^{35}$

Sometimes, the same kinds of questions were used to assess several different variables. "Engagement," "usage," and "adherence" were all assessed in terms of occurrence, duration, and frequency of use of the D-tool. Examples included asking participants how many days they used each of the pain coping skills, and counting the number of responses received to text messages. ${ }^{48,59}$

Although in some studies, defining variables and questions to assess participants' engagement in DI was difficult, others used elements from previously validated questionnaires $\left(\mathrm{eg},{ }^{33,34}\right)$. For instance, "usability" was assessed using the System Usability Scale $\left({ }^{68}\right)$ in one study. ${ }^{34}$

\subsection{3 | Results regarding engagement}

Scores for variables of engagement in Dl were high over all the 24 studies. More specifically, the scores for usability $\left(e g,{ }^{64.65}\right)$, feasibility $\left(\mathrm{eg},{ }^{35,44}\right)$, usefulness $\left(\mathrm{eg},{ }^{36,39}\right)$, and satisfaction were all high $\left(\mathrm{eg},{ }^{55,63}\right)$. Studies found that participants would recommend the D-tool tested and would continue to use it or participate again if the same DI were proposed $\left(\mathrm{eg},{ }^{36.48}\right)$. With respect to the frequency and duration of use of D-tools, participants used them more than once a week ${ }^{46}$ and adherence rates were between $70 \%$ and $100 \%\left(\mathrm{eg},{ }^{41,51}\right)$. Adherence was higher when DI included social support. ${ }^{57}$

The most popular DI functionalities in terms of higher patient engagement scores included demonstration videos and reminder features $\left(\mathrm{eg}^{33,48}\right)$. Participants mentioned that social support was the main reason to participate ${ }^{47}$ in DI. Self-monitoring, self-rating, and visual graphs were also perceived as useful, as well as the graded tasks and different stages of the exercise $\left(e g,{ }^{30.57}\right)$. Although replying to health professionals by sending messages or pictures was viewed as time consuming, it was the most-used DI feature. ${ }^{64}$ Phone calls, pedometers, and weighing scale devices were seen as very helpful to set and achieve PA and weight goals (eg, ${ }^{53.55}$ ).

Nevertheless, in two studies DI did not affect participants' engagement. $^{55,62}$ Engagement in DI varied substantially between studies. D-tools were seen as a burden and useless by some. ${ }^{36}$ Additionally, participants expressed a need for training, and for time to learn and get used to the system and its features before they could start to use it. ${ }^{64}$ Finally, participants called for the D-tools to be improved, highlighting esthetic (eg, look, comfort) and technical issues as well as difficulties they encountered when using the tools (eg, ${ }^{53,67}$ ). They also asked for more activities and media. ${ }^{51}$

\section{3 | Purposes of DI regarding psychosocial outcomes}

The most common psychosocial outcome targeted by DI was to help in the management of symptoms and pain either for patients $\left(\mathrm{eg},{ }^{30.59}\right)$ or survivors $\left(\mathrm{eg},{ }^{40.57}\right)$. Another purpose, in cancer patients only, was to improve wellbeing by targeting various factors, for instance quality of life, social support, and depression $\left\{e g,{ }^{60.61}\right.$ \} Studies about cancer survivors targeted DI focused on weight management or improvement of PA, as there is evidence that these variables play an important role in wellbeing and in avoiding relapse $\left(\mathrm{eg},{ }^{41,53}\right)$.

\section{4 | Psychosocial variables}

\subsection{1 | Measurement tools}

In the studies analyzed, the effect of DI on psychosocial variables was assessed by self-reported questionnaire. They had already been validated or used in studies previous to those analyzed here.

\subsection{2 | Psychosocial variables assessed}

Some of the studies included were designed to reduce psychological symptoms through the implementation of DI. These symptoms included sadness, distress $\left(\mathrm{eg},{ }^{38.44}\right)$, worry, ${ }^{39}$ fatigue $\left(\mathrm{eg},{ }^{29.44}\right)$, fear, ${ }^{38}$ depression, ${ }^{60}$ and anxiety and stress. ${ }^{30}$ Psychological components of pain (eg, emotional pain and pain catastrophizing) were also assessed $\left(\mathrm{eg},{ }^{39.44}\right)$.

Studies also targeted the modification of participants' perception or beliefs as a result of implementing DI. Variables included perception of social support $\left(\mathrm{eg},{ }^{31.58}\right)$, illness intrusiveness $\left(\mathrm{eg},{ }^{35.40}\right)$, perceived loss of control over eating, ${ }^{53}$ and beliefs about medication. ${ }^{36}$

In some studies, motivational variables related to pain, weight, medication adherence, and PA were measured using questionnaires 
which assessed self-efficacy $\left(\mathrm{eg}^{29.40}\right)$, motivation, basic psychological needs, and enjoyment. ${ }^{51}$ Finally, coping $\left(\mathrm{eg},{ }^{30,61}\right)$ and quality of life $\left(e g,{ }^{48.58}\right)$ were assessed.

Self-efficacy, depression, anxiety, sadness and distress, and quality of life were the most commonly examined variables. They were assessed using different scales which varied from one study to another. For example, depression was measured by using the PHQ-8 Scale $^{40}$ or the CES-D. ${ }^{60}$

\subsection{3 | Results regarding psychosocial variables}

Results regarding the efficacy of DI in changing psychosocial variables were inconsistent. Studies mentioning a positive effect of DI highlighted improvement in introjected motivation, ${ }^{51}$ and beneficial changes for symptom severity, ${ }^{62}$ fear of recurrence, helplessness, fatigue, loss of control over eating, and perceived stress $\left(\mathrm{eg}^{38,59}\right)$. In one study, social support ${ }^{58}$ and social functioning improved in both the TG and CG groups but were not statistically significant. ${ }^{63}$

In some studies. DI had a negative effect or no effect at all on psychosocial variables. For instance, three articles from one study assessed the effectiveness of a DI for medication adherence through a comparison between TG (ie, those implementing the DI) and CG The results presented in these articles were mixed. Measures of "self-efficacy" in terms of medication adherence demonstrated differences between conditions in favor of the TG in one of the articles. ${ }^{36}$ However, in other studies, these differences were not significant, ${ }^{37}$ and results were depended on recruitment criteria. ${ }^{31}$ Self-efficacy improved in two studies, ${ }^{41.53}$ and beneficial changes were also reported for perceived distress $\left(\mathrm{eg},{ }^{38.53}\right)$ and quality of life $\left(\mathrm{eg},{ }^{46,60}\right)$. However, in another study, no significant difference was observed between groups for these variables. ${ }^{59}$

Depression, stress, and illness intrusiveness were not significantly affected by DI $\left(\mathrm{eg},{ }^{35.40}\right)$. In one study, where various DI were compared with traditional in-person pain coping skills training interventions, no difference was found between both intervention strategies for effects on psychosocial variables, except for "pain catastrophizing" which decreased more in the traditional group. ${ }^{29}$ Some DI led to greater improvement in anxiety, depression score and illness intrusiveness in TG than in CG $\left(\mathrm{eg},{ }^{40.60}\right)$.

\section{5 | Results of risk of bias assessment}

The 15 RCT studies included in this review did not provide enough information about allocation concealment and blinding (Table 1 in Supporting Information). Most of these studies (9/15) were evaluated as having a high risk of bias due to the absence of blinding or missing data. No RCT study was assessed as having a low risk of bias. Non-RCT studies provided insufficient information about confounding and deviation from intended intervention (Table 2 in Supporting Information). The largest risk of bias regarded confounding, selection of participants, and missing data. No non-RCT study was assessed as having a low risk of bias. One study was assessed as having a serious risk of bias while all the others were categorized as "no information."

\section{I DISCUSSION}

This systematic review summarizes current literature on DI in the cancer setting. Twenty-nine articles were included. Results showed that DI may be effective in changing behavior and helping people cope better with disease and treatment side effects. More in-depth research is needed to have a greater understanding of DI components, participant engagement in DI, and the level to which DI may affect psychosocial variables.

The results of this review show that D-tools used in DI in the cancer context vary depending on the population targeted. Cutting-edge wearable DI to promote PA and Wi-Fi weighing scales were proposed to adolescents in one study. ${ }^{51}$ Three other studies ${ }^{53.55,63}$ which included middle-aged or elderly individuals also proposed them. In the latter, individuals over 60 years old gave a positive evaluation of $\mathrm{DI}$ and had a high score of acceptability. However, the majority of studies tested D-tools which are already part of most individuals everyday lives, (eg, text messages, computers, smartphones) precisely because they were already familiar with them. The problem with this familiarity is that DI protocols seldom envisaged training sessions to help participants use the DI more effectively as a tool which could help them cope better with their disease and treatment side effects. Indeed, some studies indicated that participants had requested training on features of existing $D 1^{30.33,60} \mathrm{It}$ is worth noting that only young people suggested improving on available DI by proposing newer DI. Older participants suggested improvements to existing DI. These results should encourage researchers to propose cutting-edge D-tools to the elderly and to provide essential training.

All the studies which assessed engagement in DI reported that participants changed their behaviors, frequently use the D-tools proposed, and expressed positive subjective experiences about them. These results confirm that DI may help improve cancer patient and survivor autonomy in symptoms management. Participants particularly appreciated visual-based characteristics of the DI analyzed, and the possibility to visually see the evolution of outcomes measured. However, social support and contact with health care providers were also important features. D-tools cannot replace human contact but are an effective complement to the interventions provided by health care professionals.

Results from the studies included in this review which examined engagement in DI showed that engagement was high. However, participants were volunteers in all cases. This means that engagement may have been overestimated. It also means that in order to encourage cancer participants' engagement in DI, they have to voluntarily choose to use D-tools to help them manage their disease and related side effects.

It is important to underline that the results about DI efficacy in changing psychosocial variables were inconsistent across the studies, with positive, negative, or no effect at all being cited. With respect to engagement, it is difficult to compare the efficacy of the DI studied because different assessment tools were used. In addition, the 24 studies analyzed in the present review varied greatly in terms of study methodology and outcome measurements. Furthermore, the DI described in the studies were diverse in terms of type and content duration and follow-up. 
This heterogeneity prevents us from identifying the most effective components of $\mathrm{DI}$ and inhibits generalization and replication. First, despite their overall common aim (to help in disease management), the DI aims differed, with some targeting psychological benefits while others focused on survivors targeted weight management and promotion of PA. It is worth noting that although the evidence for the benefits of PA during and after cancer treatment was unquestionable, ${ }^{69.70}$ it was only apparent in DI targeting breastcancer survivors (ie, women survivors). This may be due to the higher prevalence of breast cancer compared with other cancers. Nevertheless, some DI-based protocol studies targeting cancer patients (ie, both sexes, not only breast cancer) included a PA component. ${ }^{71,72}$ It is possible that interest in PA as part of cancer treatment will increase in the future. To further investigate DI effectiveness, future studies should follow this path and target diverse cancers, focusing on men as much as women, in order to allow greater comparison between studies and better understand the role of specific contexts.

Second, of the $12(12 / 24)$ studies which supposedly were theory based, only eight actually implemented the theories they mentioned in their DI analysis. Using theoretical frameworks to conceptualize, implement, and evaluate DI efficacy is critical. The lack of a theoretical framework leads to a lack of clarity around the mechanisms through which DI impact outcomes. Theories are needed to better understand the mechanisms that explain changes in behaviors, engagement, and psychosocial variables in the context of $\mathrm{Dl}$. According to Michie et $\mathrm{al}_{;}^{8}$ understanding more about human behavior could enable the development of more effective DI. Reciprocally, DI constitute unique opportunities to test and advance theories because of the type and amount of data that can be collected. ${ }^{8}$

Third, DI are multidisciplinary by nature. Expertise and collaboration from a wide range of academic disciplines, including behavioral science, computer science, and human-computer interaction, are needed. ${ }^{1.4}$ This means that DI can assess a wide range of variables in several disciplines which may have different terminology for the same variable. As shown earlier, the terminology used when assessing DI engagement can be quite confusing. There is a need to agree on a common definition of engagement, and on what psychosocial variables should be assessed and how. The studies which evaluated engagement in this review used either quantitative or qualitative methods, but not both. Future studies must use multidimensional mixed-method approaches to assessment which combine objective assessment, behaviors, and subjective experiences of users.

Furthermore, most studies (15/24) assessed engagement and psychosocial variables at the same time, but none examined the relationship between these variables. This is an important concern because engagement may affect or reflect DI efficacy on psychosocial variables, that is to say that a reciprocal relationship is as likely as an inverse relationship. For the reciprocal relationship, during $\mathrm{DI}$, the more participants are involved in DI (ie, use D-tools, express positive experience), the more they may see an improvement in psychosocial variables. Reciprocally, the more participants improve their psychosocial variables during $\mathrm{DI}$, the more they use $\mathrm{D}$-tools and express an associated positive subjective experience. Instead, for the inverse relationship, an improvement in wellibeing (eg, quality of life, motivation, self-efficacy) could lead to a decrease in the use of DI. This may be explained by the real or self-perceived development of skills and habits to deal autonomously with side effects. To increase our knowledge in the DI field, these potential reciprocal and reverse relationships need to be investigated through correlation, mediation, and moderation analysis.

As well as the diversity of studies, in terms of temporality, it is important to highlight another methodological issue which appeared. D-tools are often easily abandoned, forgotten, or lost after 6 months of use. Indeed, one study highlighted that a third of wearable fitness tracker users quit using the tool in the first 6 months after purchase. ${ }^{73}$ However, few of the studies included in our review lasted longer than 6 months. Accordingly, we have no way of knowing about behavior over the longer term. These factors need to be taken into account when interpreting results. Furthermore, future studies should last longer than 6 months and should assess follow-up evolution of outcomes several months after the end of DI. By doing so, research could enhance knowledge about how long-lasting behavioral changes really are. Similarly, our assessment of risk of bias results showed that a large number of the studies included had missing data. This high level of attrition is common in computer and internet applications. ${ }^{74}$ There is a need to examine the reasons for this in order to overcome this issue.

Finally, methodological quality was mostly poor in the studies included. The level of risk of bias varied across the studies. Primarily, there was a lack of detail that prevented the reproducibility of protocols. Additionally, the studies included had several potential sources of bias and error. In particular, missing data and the lack of blinding might have biased results. These two issues are due to the natural evolution of the use of D-tools and to the fact that loaning a D-tool to a DI participant precludes blinding. This high level of bias could explain the inconsistency in the results. Although missing data and the lack of blinding are difficult to overcome in this field of research, to increase the methodological quality of DI and allow reproducibility, future studies should at least provide more details about their protocols.

Studies on DI are difficult to implement. The lack of any observed significant difference in psychosocial variables linked to the use of D-tools could be due to the natural evolution related to treatment (eg, side effects) or recovery/remission or indeed to the fact that participants in CGs in DI have free access to digital health tools other than those proposed to the TG in the DI. There is a need to examine these variables in order to highlight the added value of DI. Additionally, a change in psychosocial variables takes time, yet DI are constrained by the general duration which participants are asked to proposed D-tools (ie, less than 6 months).

\section{1 | Study limitations}

To our knowledge, this systematic review is the first to focus on engagement with $\mathrm{DI}$ and on psychosocial variables in the cancer setting. Its main strength lies in the accuracy of the literature search through the use of a solid research strategy and strict criteria, and the use of PRISMA guidelines and Consort assessment bias tools. Nevertheless, it is not without limitations. First, it was not possible to determine DI effectiveness because of the diversity of studies, components, and measurements. Therefore, there is a need to examine the unique contribution of each of these components to DI 
effectiveness. A meta-analysis is also needed to statistically assess DI effectiveness. We could not do that here given the multiplicity of measurement tools and results displayed. Second, several studies were defined as unclear ${ }^{6}$ or as having "no information." ${ }^{13}$ Finally, only published studies were included in this review. Future reviews should include unpublished studies.

\section{2 | Clinical implications}

Digital health interventions for cancer patients and survivors show promise. They provide a unique opportunity to help patients and survivors to cope better with cancer and treatment side effects, and to enhance psychological wellbeing, leading to faster recovery. DI can complement health care providers' interventions, providing patients with tips and strategies about how to manage pain and symptoms, and how to adopt a healthier lifestyle. However, to be effective, DI need to use a theoretical framework based on behavior change theories, and last more than 6 months.

Additionally, familiarity with specific D-tools and participation of patients and survivors in developing new tools can significantly impact engagement with DI, which in turn may affect its efficacy on psychosocial variables. Accordingly, future research should consider accessing participants' habits and digital literacy and providing various ways for them to participate in the design of DI. New DI must include training sessions for patients and survivors on how to use D-tools ensure efficacy.

\section{6 | CONCLUSION}

The present review revealed that DI show promise because they enhance participants' engagement with D-tools and interventions and have positive effects on psychosocial variables and wellbeing. Nevertheless, DI require methodological and conceptual standardizations in order to be able to compare studies and better understand the mechanisms underlying participants' psychological and behavioral changes.

\section{ACKNOWLEDGEMENT}

This work was supported by the Metropole de Lyon grant

\section{ORCID}

Géraldine Escriva Boulley http://orcid.org/0000-0002-8788-2434

\section{REFERENCES}

1. Fransson P, Lund JA. Damber JE, et al. Quality of life in patients with locally advanced prostate cancer given endocrine treatment with or without radiotherapy: 4-year follow-up of SPCG-7/SFUO-3, an openlabel, randomised, phase III trial. Lancet Oncol. 2009;10(4):370-380. https://doi.org/10.1016/S1470-2045(09)70027-0

2. Janz NK, Mujahid M, Chung LK, et al. Symptom experience and quality of life of women following breast cancer treatment. J Womens Health (Larchmt). 2007;16(9):1348-1361. https://doi.org/10.1089/jwh.2006. 0255

3. Merckaert I, Libert Y, Messin S, Milani M. Slachmuylder JL, Razavi D. Cancer patients' desire for psychological support: prevalence and implications for screening patients' psychological needs. Psychooncology. 2010:19(2):141-149. https://doi.org/10.1002/pon.1568
4. Mårtensson L, Hensing G. Health literacy-a heterogeneous phenomenon: a literature review. Scand J Caring Sci. 2012;26(1):151-160. https://doi.org/10.1111/j.1471-6712.2011.00900.x

5. Riva S, Antonietti A, lannello P, Pravettoni G. What are judgment skills in health literacy? A psycho-cognitive perspective of judgment and decision-making research. Patient Pref Adher. 2015;9:1677-1686. https://doi.org/10.2147/PPA.S90207

6. Mehnert A. Employment and work-related issues in cancer survivors Critic Rev in Onco/Hemato. 2011;77(2):109-130. https://doi.org/ 10.1016/j.critrevonc.2010.01.004

7. Cooper AF, Hankins M, Rixon L, Eaton E, Grunfeld EA. Distinct workrelated, clinical and psychological factors predict return to work following treatment in four different cancer types. Psychooncology. 2013;22(3):659-667. https://doi.org/10.1002/pon.3049

8. Michie S, Yardley L, West R, Patrick K, Greaves F. Developing and evaluating digital interventions to promote behavior change in health and health care: recommendations resulting from an international workshop. J Med internet Res. 2017;19(6):e232. https://doi.org/10.2196/jmir.7126

9. Civljak M, Stead L, Hartmann-Boyce J, Sheikh A, Car \}. Internet-based interventions for smoking cessation. Cochrane Database Syst Rev. 2017;9:CD007078. https://doi.org/10.1002/14651858.CD007078. pub5

10. Nair NK, Newton NC, Shakeshaft A, Wallace P. Teesson M. A systematic review of digital and computer-based alcohol intervention programs in primary care. Curr Drug Abuse Rev. 2015;8(2):111-118.

11. Liu F, Kong $X, \mathrm{Cao}$ J, et al. Mobile phone intervention and weight loss among overweight and obese adults: a meta-analysis of randomized controlled trials. Am J Epidemiol. 2015:181(5):337-348. https://doi. org/10.1093/aje/kwu260

12. Muntaner A. Vidal-Conti J, Palou P. Increasing physical activity through mobile device interventions: a systematic review. Health Informatics J. 2015;22(3):451-469. https://doi.org/10.1177/1460458214567004

13. Jones KR, Lekhak $N$, Kaewluang $N$. Using mobile phones and short message service to deliver self-management interventions for chronic conditions: a meta-review. Worldviews on Ev-Based Nurs. 2014:11(2):81-88. https://doi.org/10.1111/wvn.12030

14. Perski O, Blandford A, West R, Michie S. Conceptualising engagement with digital behaviour change interventions: a systematic review using principles from critical interpretive synthesis. Transl Behav Med 2017;7(2):254-267. https://doi.org/10.1007/s13142-016-0453-1

15. Donkin L, Christensen H, Naismith SL, Neal B, Hickie IB, Glozier N. A systematic review of the impact of adherence on the effectiveness of e-therapies. J Med internet Res. 2011;13(3):e52. https://doi.org/ 10.2196/jmir.1772

16. Cobb NK, Graham AL, Bock BC, Papandonatos G, Abrams DB. Initial evaluation of a real-world internet smoking cessation system. Nicotine Tob Res. 2005;7(2):207-216. https://doi.org/10.1080/ 14622200500055319

17. Tate DF, Wing RR, Winett R. Using internet technology to deliver a behavioral weight loss program. JAMA. 2001:285(9):1172-1177. https://doi.org/10.1001/jama.285.9.1172

18. Wesley KM, Fizur PJ. A review of mobile applications to help adolescent and young adult cancer patients. Adolesc Health Med Ther. 2015;6:141-148. https://doi.org/10.2147/AHMT.S69209

19. Odeh B, Kayyali R, Nabhani-Gebara S, Philip N. Optimizing cancer care through mobile health. Support Care Cancer. 2015;23(7):2183-2188. https://doi.org/10.1007/s00520-015-2627-7

20. Goode AD, Lawler SP, Brakenridge CL, Reeves MM, Eakin EG. Telephone, print, and web-based interventions for physical activity, diet, and weight control among cancer survivors: a systematic review. $J$ Cancer Surviv. 2015;9(4):660-682. https://doi.org/10.1007/s11764-0150442-2

21. Hong Y, Peña-Purcell NC, Ory MG. Outcomes of online support and resources for cancer survivors: a systematic literature review. Patient Educ Couns. 2012;86(3):288-296. https://doi.org/10.1016/j.pec.2011. 06.014 
22. Seiler A, Klaas V, Tröster G, Fagundes CP. eHealth and mHealth interventions in the treatment of fatigued cancer survivors: a systematic review and meta-analysis. Psychooncology. 2017;26(9):1239-1253. https://doi.org/10.1002/pon.4489

23. Kuijpers W, Groen WG, Aaronson NK, Van Harten WH. A systematic review of web-based interventions for patient empowerment and physical activity in chronic diseases: relevance for cancer survivors. J Med Internet Res. 2013;15(2):e37. https://doi.org/10.2196/ jmir.2281

24. Kopp LM, Gastelum Z, Guerrero CH, Howe CL, Hingorani P, Hingle M. Lifestyle behavior interventions delivered using technology in childhood, adolescent, and young adult cancer survivors: a systematic review. Pediatr Blood Cancer. 2017;64(1):13-17. https://doi.org/ $10.1002 /$ pbc. 26166

25. Shea BJ, Grimshaw JM, Wells GA, et al. Development of AMSTAR: a measurement tool to assess the methodological quality of systematic reviews. BMC Med Res Methodol. 2007;7(1):10. https://doi.org/ 10.1186/1471-2288-7-10

26. Shea BJ, Hamel C, Wells GA, et al. AMSTAR is a reliable and valid measurement tool to assess the methodological quality of systematic reviews. J Clin Epidemiol. 2009;62(10):1013-1020. https://doi.org/ 10.1016/j.jclinepi.2008.10.009

27. Higgins JPT, Altman DG, Gotzsche PC, et al. The Cochrane Collaboration's tool for assessing risk of bias in randomised trials. BMJ 2011;343(oct18 2):d5928. https://doi.org/10.1136/bmj.d5928

28. Sterne JA, Hernán MA, Reeves BC, et al. ROBINS-I: a tool for assessing risk of bias in non-randomised studies of interventions. BMJ. 2016:355:i4919. https://doi.org/10.1136/bmj.i4919

29. Somers TJ, Kelleher SA, Westbrook KW, et al. A small randomized controlled pilot trial comparing mobile and traditional pain coping skills training protocols for Cancer patients with pain. Pain Res Treat. 2016;2016:1-8. https://doi.org/10.1155/2016/2473629

30. Sundberg K. Wengstrom Y, Blomberg K, Halleberg-Nyman M, Frank C Langius-Eklof A. Early detection and management of symptoms using an interactive smartphone application (Interaktor) during radiotherapy for prostate cancer. Support Care Cancer. 2017;25(7):2195-2204. https://doi.org/10.1007/s00520-017-3625-8

31. Spoelstra SL, Sikorskii A, Given CW, et al. Adult Cancer Patient Recruitment and Enrollment into Cell Phone Text Message Trials. Telemed e-Health. 2016;22(10):836-842. https://doi.org/10.1089/ tmj.2015.0263

32. Foley NM, O'Connell EP, Lehane EA, et al. PATI: Patient accessed tailored information: A pilot study to evaluate the effect on preoperative breast cancer patients of information delivered via a mobile application. Breast. 2016;30:54-58. https://doi.org/10.1016/j.breast.2016.08.012

33. Fu MR, Axelrod D, Guth $A A$, et al. mHealth self-care interventions: managing symptoms following breast cancer treatment. mHealth. 2016;2:28. https://doi.org/10.21037/mhealth.2016.07.03

34. Short C, Rebar A, James E, et al. How do different delivery schedules of tailored web-based physical activity advice for breast cancer survivors influence intervention use and efficacy? J Cancer Surviv. 2017;11(1):80-91. https://doi.org/10.1007/s11764-016-0565-0

35. Classen CC, Chivers ML. Urowitz S, et al. Psychosexual distress in women with gynecologic cancer: A feasibility study of an online support group. Psychooncology. 2013;22(4):930-935. https://doi.org/ 10.1002/pon.3058

36. Spoelstra S, Given C, Sikorskii A, et al. Feasibility of a Text Messaging Intervention to Promote Self-Management for Patients Prescribed Oral Anticancer Agents. Oncol Nurs Forum. 2015;42(6):647-657. https://doi.org/10.1188/15.ONF.647-657

37. Spoelstra SL, Given CW, Sikorskii A, et al. Proof of Concept of a Mobile Health Short Message Service Text Message Intervention That Promotes Adherence to Oral Anticancer Agent Medications: A Randomized Controlled Trial. Telemed e-Health. 2016;22(6):497-506. https://doi.org/10.1089/tmj.2015.0126
38. Van Den Berg SW, Gielissen MFM, Custers JAE, Van Den Graaf WTA, Ottevanger PB, Prins JB. BREATH: Web-based self-management for psychological adjustment after primary breast cancer-results of a multicenter randomized controlled trial. J Clin Oncol. 2015;33(25): 2763-2771. https://doi.org/10.1200/JCO.2013.54.9386

39. Fortier MA, Chung WW, Martinez A, Gago-Masague S, Sender L. Pain buddy: A novel use of $m$-health in the management of children's cancer pain. Comput Biol Med. 2016:76:202-214. https://doi.org/10.1016/j. compbiomed.2016.07.012

40. Chen Z, Koh PW, Ritter PL, Lorig K, Bantum EO, Saria S. Dissecting an online intervention for cancer survivors: Four exploratory analyses of internet engagement and its effects on health status and health behaviors. Heal Educ Behav Off Publ Soc Public Heal Educ. 2015;42(1):32-45. https://doi.org/10.1177/1090198114550822

41. McCarroll ML, Armbruster S, Pohle-Krauza RJ, et al. Feasibility of a lifestyle intervention for overweight/obese endometrial and breast cancer survivors using an interactive mobile application. Gynecol Oncol. 2015;137(3):508-515. https://doi.org/10.1016/j.ygyno.2014.12.025

42. Bandura A. Self-efficacy: Toward a unifying theory of behavioral change. Psychol Rev. 1977;84:191-215.

43. Bandura A. Self Efficacy: the exercise of control. Am J Health Promot. 1997;149(3):8-10

44. Somers TJ, Abernethy AP, Edmond SN, et al. A Pilot Study of a Mobile Health Pain Coping Skills Training Protocol for Patients with Persistent Cancer Pain. J Pain Symptom Manag. 2015;50(4):553-558. https://doi. org/10.1016/j.jpainsymman.2015.04.013

45. Melzack R, Wall PD. Pain Mechanisms: A New Theory. Science. 1965:150(3699):971-979

46. Hong YA. Goldberg D, Ory MG, et al. Efficacy of a Mobile-Enabled Web App (iCanFit) in Promoting Physical Activity Among Older Cancer Survivors: A Pilot Study. JMIR Cancer. 2015;1(1):e7. https://doi.org/ 10.2196/cancer.4389

47. Locke EA, Latham GP. Building a practically useful theory of goal setting and task motivation: A 35-year odyssey. Am Psychol. 2002;57(9):705-717. https://doi.org/10.1037//0003-066X.57.9.705

48. Forbes CC, Blanchard CM, Mummery WK, Courneya KS. Feasibility and Preliminary Efficacy of an Online Intervention to Increase Physical Activity in Nova Scotian Cancer Survivors: A Randomized Controlled Trial. JMIR Cancer. 2015;1(2):e12. https://doi.org/10.2196/cancer. 4586

49. Prochaska JO, Velicer WF. The Transtheoretical Model of Health Behaviour Change. Am J Health Promot. 1997;12(1):38-48.

50. Ajzen I. The theory of planned behavior. Organ Behav Hum Decis Process. 1991;50(2):179-211.

51. Mendoza JA. Baker KS, Moreno MA, et al. A Fitbit and Facebook mHealth intervention for promoting physical activity among adolescent and young adult childhood cancer survivors: A pilot study. Pediatr Blood Cancer. 2017;64(12):e26660. https://doi.org/10.1002/ pbc. 26660

52. Deci EL, Ryan RM. Self-determination theory: A macrotheory of human motivation, development, and health. Can Psychol Can. 2008;49(3):182-185. https://doi.org/10.1037/a0012801

53. Quintiliani L. Mann DM, Puputti M, Quinn E, Bowen DJ. Pilot and Feasibility Test of a Mobile Health-Supported Behavioral Counseling Intervention for Weight Management Among Breast Cancer Survivors. JMIR Cancer. 2016;2(1):e4. https://doi.org/10.2196/cancer.5305

54. Sorensen G, Emmons K, Hunt MK, et al. Model for incorporating social context in health behavior interventions: Applications for cancer prevention for working-class, multiethnic populations. Prev Med (Baltim). 2003;37(3):188-197. https://doi.org/10.1016/S0091-7435(03)00111-7

55. Valle CG, Deal AM. Tate DF. Preventing weight gain in African American breast cancer survivors using smart scales and activity trackers: a randomized controlled pilot study. J Cancer Surviv. 2017;11(1):133-148. https://doi.org/10.1007/s11764-016-0571-2 
56. Kanfer FH. Self-regulation: Research, issues, and speculations. Behav Modif Clin Psychol. 1970;74:178-220.

57. Duffecy J, Sanford S, Wagner L, Begale M, Nawacki E, Mohr DC Project onward: An innovative e-health intervention for cancer survivors. Psychooncology. 2013;22(4):947-951. https://doi.org/10.1002/ pon. 3075

58. Gustafson DH, Hawkins R, Pingree S, et al. Effect of computer support on younger women with breast cancer. J Gen Intern Med. 2001;16(7):435-445. https://doi.org/10.1046/j.1525-1497.2001 016007435.x

59. Ruland CM, Andersen T, Jeneson A, et al. Effects of an internet support system to assist cancer patients in reducing symptom distress: A randomized controlled trial. Cancer Nurs. 2013;36(1):6-17. https://doi. org/10.1097/NCC.0b013e31824d90d4

60. Lieberman MA, Goldstein BA. Not all negative emotions are equal: The role of emotional expression in online support groups for women with breast cancer. Psychooncology. 2006;15(2):160-168. https://doi.org/ 10.1002/pon.932

61. Winzelberg AJ, Classen C, Alpers GW, et al. Evaluation of an internet support group for women with primary breast cancer. Cancer 2003;97(5):1164-1173. https://doi.org/10.1002/cncr.11174

62. Spoelstra SL, Given BA, Given CW, et al. An intervention to improve adherence and management of symptoms for patients prescribed ora chemotherapy agents: An exploratory study. Cancer Nurs. 2013;36(1):18-28. https://doi.org/10.1097/NCC.0b013e3182551587

63. Uhm KE, Yoo JS, Chung SH, et al. Effects of exercise intervention in breast cancer patients: is mobile health (mHealth) with pedometer more effective than conventional program using brochure? Breast Cancer Res Treat. 2017:161(3):443-452. https://doi.org/10.1007/s10549016-4065-8

64. Mirkovic J, Kaufman DR, Ruland CM. Supporting cancer patients in illness management: usability evaluation of a mobile app. JMiR mHealth uHealth. 2014;2(3):e33. https://doi.org/10.2196/mhealth.3359

65. Hochstenbach LMJ, Zwakhalen SMG, Courtens AM, van Kleef M, de Witte LP. Feasibility of a mobile and web-based intervention to support self-management in outpatients with cancer pain. Eur J Oncol Nurs. 2016;23:97-105. https://doi.org/10.1016/j.ejon.2016.03.009

66. Puszkiewicz P, Roberts AL, Smith L, Wardle J, Fisher A. Assessment of Cancer Survivors' Experiences of Using a Publicly Available Physical Activity Mobile Application. JMIR Cancer. 2016;2(1):e7. https://doi. org/10.2196/cancer.5380

67. Harder $\mathrm{H}$, Holroyd P, Burkinshaw L, et al. A user-centred approach to developing bWell, a mobile app for arm and shoulder exercises after breast cancer treatment. J Cancer Surviv. 2017;11(6):732-742. https://doi.org/10.1007/s11764-017-0630-3

68. Brooke J. System Usability Scale (SUS): A Quick-and-Dirty Method of System Evaluation User Information. In: Jordan PW, Thomas B,
Weerdmeester BA, McClelland IL, eds. Usability Evaluation in Industry. London: Taylor and Francis; 1996:189-194.

69. Doyle C, Kushi LH, Byers T, Courneya KS, Demark-Wahnefried W. Grant B, McTiernan A, Rock CL, Thompson C, Gansler T, Andrews KS, 2006 Nutrition, Physical Activity and Cancer Survivorship Advisory Committee., American Cancer Society.. Nutrition and physical activity during and after cancer treatment: an American Cancer Society guide for informed choices. CA Cancer J Clin.;56(6):323-53. https://doi.org/ 10.3322/canjclin.56.6.323

70. Knols R, Aaronson NK, Uebelhart D, Fransen J, Aufdemkampe G. Physical exercise in cancer patients during and after medical treatment: systematic review of randomized and controlled clinical trials. J Clin Oncol. 2005:23(16):3830-3842. https://doi.org/10.1200/JCO.2005 02.148

71. Agboola S, Flanagan C, Searl M, Elfiky A, Kvedar J, Jethwani K. Improving Outcomes in Cancer Patients on Oral Anti-Cancer Medications Using a Novel Mobile Phone-Based Intervention: Study Design of a Randomized Controlled Trial. JMIR Res Protoc. 2014;3(4):e79. https://doi.org/10.2196/resprot.4041

72. Fishbein JN, Nisotel LE, MacDonald JJ, et al. Mobile Application to Promote Adherence to Oral Chemotherapy and Symptom Management: A Protocol for Design and Development. JMIR Res Protoc 2017;6(4):e62. https://doi.org/10.2196/resprot.6198

73. Hermsen S, Frost J, Renes RJ, Kerkhof P. Using feedback through digital technology to disrupt and change habitual behavior: A critical review of current literature. Comput Hum Behav. 2016:57:61-74. https://doi.org/10.1016/j.chb.2015.12.023

74. Tate DF, Zabinski MF. Computer and Internet Applications fo Psychological Treatment: Update for Clinicians. J Clin Psychol 2004;60(2):209-220. https://doi.org/10.1002/jclp.10247

\section{SUPPORTING INFORMATION}

Additional supporting information may be found online in the Supporting Information section at the end of the article.

How to cite this article: Escriva Boulley G, Leroy $T$, Bernetière C, Paquienseguy F, Desfriches-Doria O, Préau M. Digital health interventions to help living with cancer: A systematic review of participants' engagement and psychosocial effects. Psycho-Oncology. 2018;1-10. https://doi.org/ $10.1002 /$ pon.4867 